\title{
High-power operation of coherently coupled tapered laser diodes in an external cavity
}

\author{
G. Schimmel a, I. Doyen ${ }^{\text {a }}$, S. Janicot ${ }^{\text {a }}$, M. Hanna a , P. Georges ${ }^{\text {a }}$, G. Lucas-Leclin ${ }^{* a}$, J. Decker ${ }^{\text {b }}$, \\ P. Crump ${ }^{\text {b }}$, G. Erbert ${ }^{\text {b }}$, S. Kaunga-Nyirenda ${ }^{\text {c }}$, D. Moss ${ }^{\text {c }}$, S. Bull ${ }^{\text {c }}$, E. C. Larkins ${ }^{\text {c }}$, \\ U. Witte ${ }^{\mathrm{d}}$, M. Traub ${ }^{\mathrm{d}}$ \\ a Laboratoire Charles Fabry, Institut d'Optique Graduate School, CNRS, Université Paris Saclay, \\ Palaiseau, France; \\ ${ }^{\mathrm{b}}$ Ferdinand-Braun-Institut, Leibniz-Institut für Höchstfrequenztechnik, Berlin, Germany; \\ ${ }^{\mathrm{c}}$ School of Electrical and Electronic Engineering, University of Nottingham, Nottingham, U.K; \\ ${ }^{\mathrm{d}}$ Fraunhofer-Institut fur Lasertechnik, Aachen, Germany.
}

*gaelle.lucas-leclin@institutoptique.fr

\begin{abstract}
We demonstrate a rear-side phase-locking architecture with two high-brightness diode lasers. This technique is based on the passive phase-locking of emitters in an external cavity on their rear facet, and their coherent combination on the front facet. Two high-brightness high-power tapered laser diodes are coherently combined using a Michelson-based cavity. The combining efficiency is above $80 \%$ and results in an output power of $6.7 \mathrm{~W}$ in a nearly diffraction-limited beam. The rear-side architecture is then used with a laser bar of 5 tapered emitters using an interferometric extended cavity, based on a diffractive optical element. We describe the experimental evaluation of the diffractive optical element, and the phase-locked operation of the laser bar.
\end{abstract}

Keywords: coherent beam combining, passive phase-locking, self-organized extended cavity, diode lasers

\section{INTRODUCTION}

Diode lasers are the most efficient technology for converting electrical energy into useful light. However, this efficiency is not available to most industrial users due to the low brightness of direct diode laser sources. Overcoming this limitation would deliver a technological breakthrough in cost effective, high-brilliance laser diode sources for industrial applications. The beam combination of several high-brilliance diode sources appears to be the best option to that goal. Industrial diode laser systems based on spectral beam combining (SBC) or incoherent beam combining (IBC) are already available ${ }^{1,2}$. Combination of SBC and IBC can also be implemented with the aim to reach the $\mathrm{kW}$ level of continuous optical power with a good beam quality. Such systems would consist of several stages involving successive beam combination set-ups: this is the architecture investigated within the European-funded BRIDLE project ${ }^{3}$. The building block of these systems is based on low power diode laser emitters with a very good beam quality and a high electrical-tooptical (E-O) conversion efficiency. Further scaling of the final output brightness would imply to improve the initial brightness of the building block, for example by the use of coherent beam combining (CBC) techniques. CBC consists in the superposition of individual laser beams by constructive interference, providing a single high-power laser beam with excellent spectral and spatial properties ${ }^{4}$. Over the years, different approaches were investigated ${ }^{5}$ : either active phaselocking of amplifiers ${ }^{6,7}$ or passive self-organization of emitters in a common laser cavity ${ }^{8-12}$. For simplicity and industrial purposes, passive techniques are here favored to improve the building block performance.

In the following, we investigate a new $\mathrm{CBC}$ architecture using a common extended cavity on the back side of diode lasers for phase locking, while the coherent beam superposition of the phase-locked beams is realized on the front side. As a result, the E-O conversion efficiency of the phase-locked laser cavity is increased as compared to standard frontside configurations ${ }^{9-11}$. Moreover, such an extended cavity placed on the rear side provides the strong optical feedback required for phase-locked operation far from the laser threshold. This configuration is first demonstrated with two 
individual high-brightness tapered devices ${ }^{13}$ in a Michelson-type extended cavity ${ }^{12,14}$, highlighting the capability of such setup for high power operation. A cavity architecture is then proposed to extend this configuration to a 5 -emitter laser bar, with the use of a continuous phase diffractive optical element as the combiner element. The experimental evaluation of the phase grating is achieved and the phase-locked operation of the laser bar is studied.

\section{PHASE LOCKING AND COHERENT COMBINING OF TWO EMITTERS}

\subsection{Description of the tapered lasers}

The tapered laser devices used emitted at around $\lambda=976 \mathrm{~nm}$. The lasers consist of a $2 \mathrm{~mm}$ long ridge section, and a 4 $\mathrm{mm}$ long tapered section $\left(\alpha_{\mathrm{T}}=6^{\circ}\right.$ taper angle). The front (tapered section) facet has a $0.5 \%$ reflectivity, while the rear (ridge section) facet has a $\mathrm{R}<0.1 \%$ coating. Details of the design of the tapered diodes are given in [ref. 15]. The tapered lasers are mounted p-side up on C-mounts whose dimension matches to the total chip length of $6 \mathrm{~mm}$, in order to allow optical access to both facets. As compared to conductively cooled packages, it limits how effectively the lasers are cooled. Electrical contact to the tapered region is made via a custom-dimensioned $\mathrm{CuW}$ sub-mount, which is soldered to the $\mathrm{p}$-side of the device for uniform pumping and improved cooling. The ridge section is contacted separately via wirebonds. The two sections are separately driven by currents $\mathrm{I}_{R}$ and $\mathrm{I}_{\mathrm{T}}$, respectively. With a high-reflectivity extended cavity on their rear facet, the extracted optical power reaches $4 \mathrm{~W}$ at $\mathrm{I}_{\mathrm{R}}=400 \mathrm{~mA}$ and $\mathrm{I}_{\mathrm{T}}=6 \mathrm{~A}$, corresponding to an E-O conversion efficiency of $33 \%$. The beam is diffraction-limited along the fast axis, while along the slow axis, the beam quality factor is $\mathrm{M}_{4 \sigma}^{2} \approx 2.5$ at $\mathrm{I}_{\mathrm{T}}=6 \mathrm{~A}$ - with about $80 \%$ of the extracted power contained in the diffraction-limited central lobe. The radiance, that highlights the ability of extracting high optical power $\mathrm{P}$ while maintaining a good beam quality, is $\mathrm{B}=\mathrm{P} /\left(\lambda^{2} \times \mathrm{M}^{2}\right) \approx 200 \mathrm{MW} \cdot \mathrm{cm}^{-2} \cdot \mathrm{sr}^{-1}$ at $6 \mathrm{~A}$. For such lasers, separate (rear side) phase-locking and (front side) coherent combining is the preferred and most promising configuration, as optical feedback into the front facet (tapered-section) might deteriorate the beam quality, and even lead to early device failure. Additionally the phaselocking is achieved with diffraction limited beams from the rear (ridge) sections.

First, the phase noise characterization of the devices is done using a Mach-Zehnder interferometer as follows: a seed laser beam is split into two arms, one including a tapered amplifier device and the other acting as a reference arm; the interference pattern is then observed after superposition of both beams. The phase-shift induced by propagation through the amplifier is measured as a function of the operating currents in the ridge section. The single-pass phase shift is typically equal to $\pi / 35$ per $\mathrm{mA}$ of the ridge current (see Fig. 1, left). Since gain is saturated in the tapered section, the optical power extracted from each device remains nearly unaffected through a phase tuning of $2 \pi$ with $\mathrm{I}_{R}$. Additionally, time fluctuations of the phase accumulated through an emitter are measured using both outputs I (in-phase) and Q (quadrature) of the interferometer: the phase is unambiguously determined by computing the inverse tangent of the ratio of Q and I. The resulting phase-noise, shown in Figure 1 (right), exhibits a low level in the high frequency range. As a result, the integrated phase noise shows that the most important contribution is below $10 \mathrm{~Hz}$ - indicating that the largest contribution comes from thermal drifts, with faster fluctuations only contributing to an integrated phase noise below $\pi / 100$.
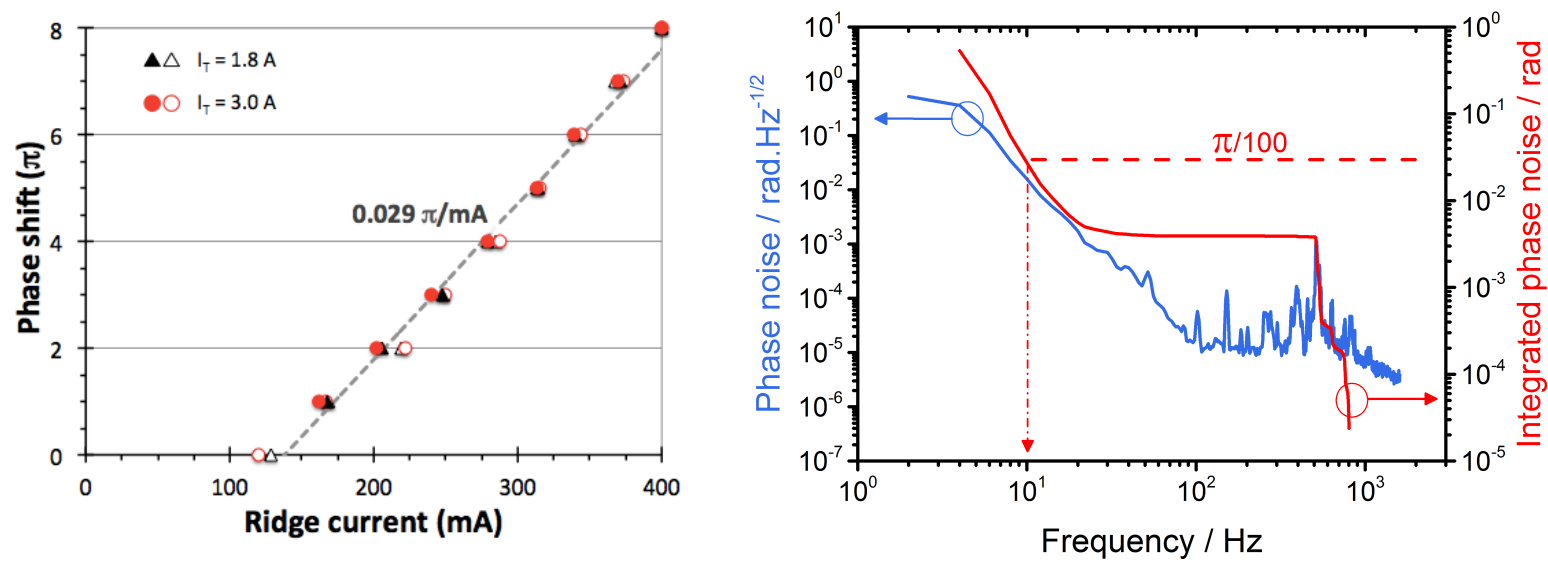

Figure 1. Phase shift induced as a function of the ridge section current at two different tapered section current (left); phase noise and integrated phase noise of a tapered amplifier (right). 


\subsection{Experimental set-up: rear-side Michelson external cavity}

We now describe the phase locking architecture with two emitters. The extended cavity is based on a Michelson interferometer on the rear side featuring an external reflector. The front facets of each diode are the output couplers of the laser cavity, coated to yield $0.5 \%$ reflectivity which is sufficient to achieve lasing owing to the high gain in the diode medium. The cavity back-end features an external 2000 lines/mm diffraction grating (at Littrow incidence), which serves as a common, dispersive high reflector and controls the wavelength (see Fig. 2). This ensures a stable and narrow laser line at $976 \mathrm{~nm}$. Both laser beams are collimated with high NA aspheric lenses $\left(\mathrm{F}_{1} \& \mathrm{~F}_{2}\right)$ and combined on a 50/50 beamsplitter $\left(\mathrm{BS}_{1}\right)$. Since both lasers share the same extended cavity, they undergo minimum losses if the beams are in phase at $\mathrm{BS}_{1}$ - resulting in constructive interference on the $\mathrm{P}$ arm and destructive interference on the other arm. On the other hand, incoherent operation of both lasers induces $75 \%$ losses per roundtrip in the cavity for each laser. Coherent operation is thus strongly favored by the Michelson extended cavity.

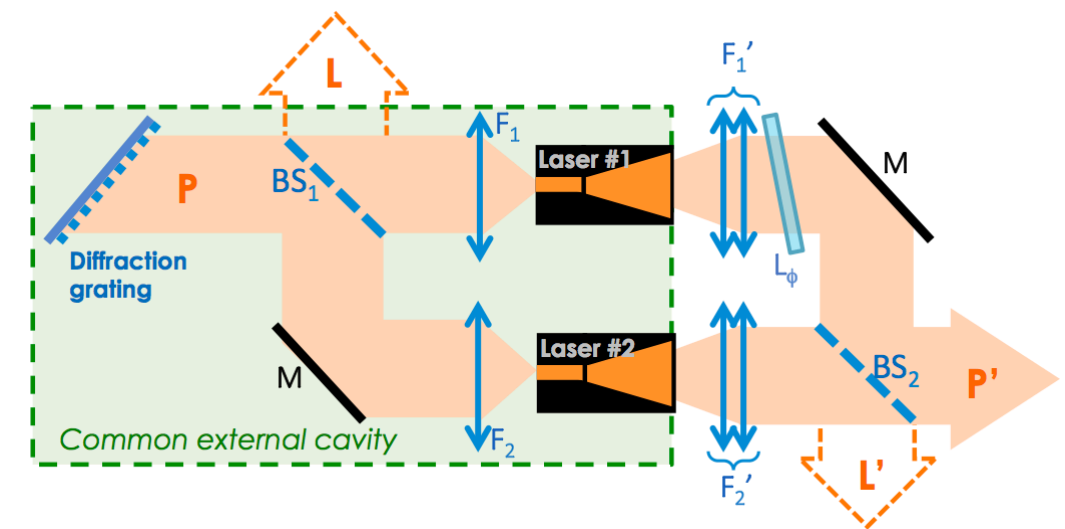

Figure 2. Experimental set-up with the rear-side Michelson extended cavity and the front-side coherent superposition; $\mathrm{BS}_{1-2}: 50 / 50$ beamsplitters; P, P': useful arms; L, L': losses. ; $F_{1,2}: 8 \mathrm{~mm}$ aspheric lenses; $F_{1}{ }_{2}, 2$ : combination of $2.75 \mathrm{~mm}$ aspheric lenses and $19 \mathrm{~mm}$ cylindrical lenses; M: high reflective mirrors.

On the taper (front) side, an arrangement of an aspheric lens and a cylindrical lens $\left(\mathrm{F}_{1}^{\prime} \& \mathrm{~F}_{2}^{\prime}\right)$ are used to correct for the intrinsic astigmatism of the tapered laser devices, and a simple 50/50 beamsplitter $\left(\mathrm{BS}_{2}\right)$ is used as a combiner to perform coherent superposition of the beams. A phase plate $\mathrm{L}_{\Phi}$ is added on one arm to adjust the phase relationship between the two laser beams, as their optical paths are different. It is a simple anti-reflection coated 0.5-mm thick plane silica plate, whose rotation allows fine tuning of the phase difference and maximizing of the combined power in the $\mathrm{P}^{\prime}$ arm. The common, back-end external grating (which, along with each emitter front facet, forms the two laser cavities) performs the phase-locking function. From $\mathrm{BS}_{1}$ to the rear facets of each emitter, the arms are 25 and $160 \mathrm{~mm}$ long respectively.

\subsection{Phase-locking and coherent combining}

The passive phase-locking operation of the two lasers is realized for currents up to $\mathrm{I}_{\mathrm{T}}=6 \mathrm{~A}$, which is as high as 5 times the laser threshold. On the front side, the laser power extracted from each device is $\mathrm{P}_{1}=\mathrm{P}_{2} \approx 4 \mathrm{~W}$ at $\mathrm{I}_{\mathrm{R}}=400 \mathrm{~mA}$ and $\mathrm{I}_{\mathrm{T}}$ $=6 \mathrm{~A}$ in phase-locked operation. The maximum combined optical power $\mathrm{P}^{\prime}$ (see Fig. 2) is $6.7 \mathrm{~W}$, which corresponds to a net efficiency, defined as $\eta_{\mathrm{P}}^{\prime},=\mathrm{P}^{\prime} /\left(\mathrm{P}_{1}+\mathrm{P}_{2}\right)$, in excess of $82 \%$. By this definition, this figure also accounts for all loss mechanisms inherent in our system.

Experimentally, we observe that the extended cavity acts as a lateral mode filter: the slow axis (SA) beam quality for each emitter is improved to $\mathrm{M}_{4 \sigma}^{2} \leq 1.3$ when both emitters are phase-locked at $\mathrm{I}_{\mathrm{T}}=6 \mathrm{~A}$ (see Fig. 3b). Since the combining stage on the front facets operates as a second spatial filter, the beam quality of the combined beam is further enhanced to $\mathrm{M}^{2}{ }_{4 \sigma} \leq 1.2$ (see Fig. 3d). Indeed, only the common features in the spatial intensity and phase of both beams are coherently combined. This results in a spatial cleaning of the combined beam, whereby lateral modes are rejected on the loss arm L'. Thus the radiance of the laser system is enhanced to B $\approx 500 \mathrm{MW} . \mathrm{cm}^{-2} . \mathrm{sr}^{-1}$, with $6.7 \mathrm{~W}$ of combined power. The corresponding E-O conversion efficiency is $27 \%$, higher than what is achieved in standard front side extended cavities $^{9-11}$ owing to the low reflectivity coatings on the front facets, which is made possible by our rear-side architecture. 
(a)

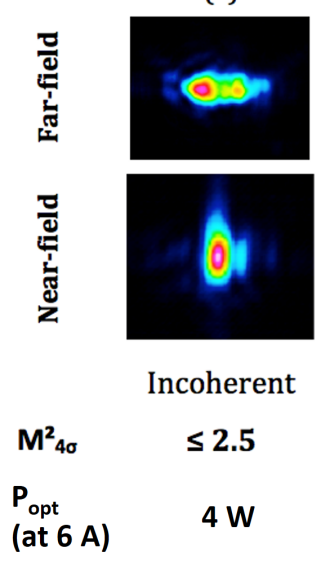

(b)

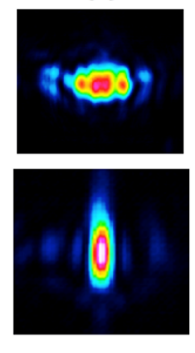

Phase-locked

$\leq 1.3$

$4 \mathrm{~W}$ (c)

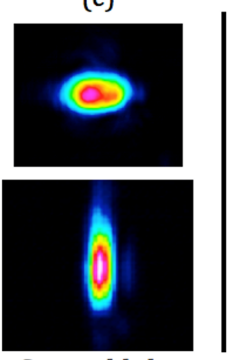

Central lobe (phase-locked) $\leq \mathbf{1 . 0 5}$

$3.2 \mathrm{~W}$ (d)

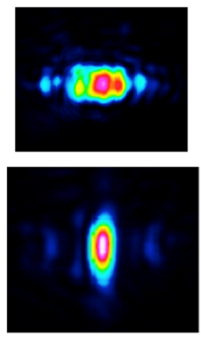

Combined

$\leq 1.2$

Figure 3. Intensity profiles of the far field (top) and near field (bottom) for laser 1 alone (a, b, c) and the combined output (d). Vertical and horizontal directions correspond respectively to the fast and slow axes. The $\mathrm{M}^{2}$ values are given for the slow axis.

The combining efficiency is limited by several factors, such as the proportion of incoherent light or mismatches between the beams ${ }^{16}$. To illustrate the high coherence between beams to be combined, a two-wave interference pattern is obtained by crossing the beams at a large angle (see Fig. 4). In the central part of the beams, dominated by the fundamental mode content, the visibility is $96 \%$ and can be shown to be a lower bound of the mutual coherence ${ }^{17}$. This is an indication that the ASE of the tapered devices is not a limitation, which is further confirmed by the spectrum analysis of the extendedcavity laser emission.

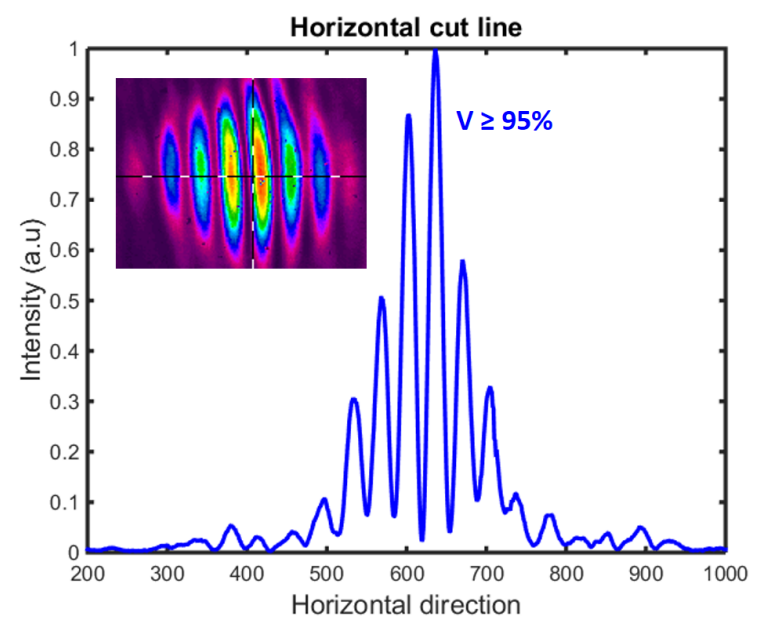

Figure 4. Transverse profile of the interference between the two laser beams from the front side of the tapered emitters under phaselocked operation at $6 \mathrm{~A}$, in the slow-axis direction; the value of the visibility is given for the center of the pattern.

Thus the remaining origins of the decrease in the beam combining efficiency come from mismatches in intensity and phase profiles. Since the beams are nearly single transverse mode, these mismatches can be separated in two parts: differences in higher-order mode content, and differences in fundamental mode profiles. To identify these contributions, first the intensity mismatches for the full beams are quantified from the overlap calculation between both measured intensity profiles ${ }^{18}$ leading to $9 \%$ reduction of the combining efficiency at $\mathrm{I}_{\mathrm{T}}=6 \mathrm{~A}$. Second, we isolate the fundamental mode content by implementing a spatial filtering stage on the combined arm P' for the slow-axis (SA). The central diffraction-limited lobe of the beams is selected, corresponding to $80 \%$ of the optical output power for each beam (Fig. $3 \mathrm{c})$. Then the combined optical power reaches $6 \mathrm{~W}$ in a purely Gaussian beam, indicating that the combining efficiency is significantly improved from $82 \%$ to $92 \%$ at $\mathrm{I}_{\mathrm{T}}=6 \mathrm{~A}$ and up to $95 \%$ at $3 \mathrm{~A}$. These results are summarized in Figure 5 . A $5 \%$ decrease in efficiency in this case can be identified as a consequence of a residual astigmatism difference between 
fundamental modes. It is noteworthy that the output power of $6 \mathrm{~W}$, obtained behind the SA filtering stage, demonstrates that $89 \%$ of the combined power extracted directly from the laser system is actually into the diffraction-limited central lobe. With this SA filtering, the radiance of our laser system reaches $600 \mathrm{MW} \cdot \mathrm{cm}^{-2} \cdot \mathrm{sr}^{-1}$.
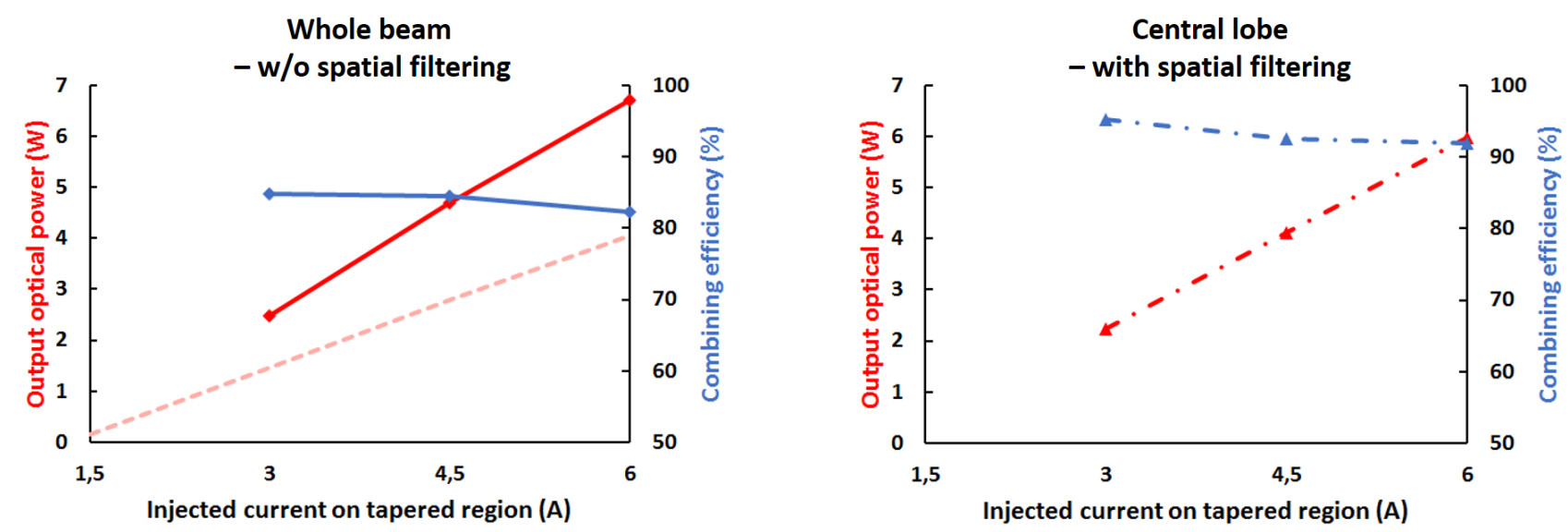

Figure 5. Extracted optical power and corresponding combining efficiency from the experimental set-up on arm P' w/o any spatial filtering stage (left) and for the filtered central lobe (right) as a function of the injected current on the tapered region. The light red dotted line on the right corresponds to the power extracted from a single individual emitter operating in an extended-cavity.

\section{EXTENSION TO A FIVE-EMITTER LASER BAR}

\subsection{Laser bar}

The following experiment is realized using a 5-tapered-emitter laser bar. The tapered laser devices used are similar to the individual ones described previously, with the same reflective coatings on both facets. The laser bar is soldered on a specifically designed copper heat sink to allow access to both facets while ensuring a good thermal evacuation. Each emitter is separately driven by both $I_{R}$ and $I_{T}$ currents (see Fig. 6), several wires are soldered from the edge contacts to the upper p-region of the emitters to ensure uniform electrical pumping. The collimation on the fast-axis is ensured by a cylindrical fast-axis collimator (FAC) with a focal length of $0.6 \mathrm{~mm}$ and a numerical aperture of 0.8 . The smile of the laser bar has been measured below $0.5 \mu \mathrm{m}$ RMS on both front and rear sides. Without any extended cavity, only spontaneous emission from the emitters is observed up to $3.5 \mathrm{~A}$, showing the good quality of the AR coating on their rear (ridge section) facet.

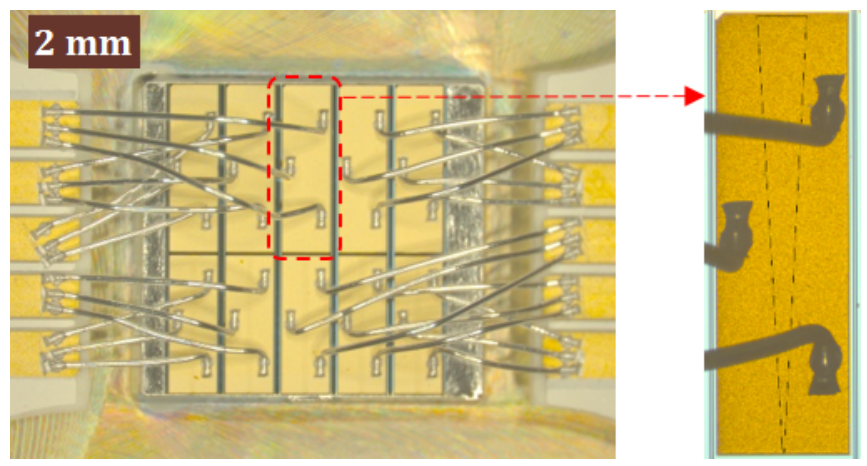

Figure 6. Picture of the 5-emitter tapered laser bar (top view), the front (tapered) section is located on the top of the picture while the rear (ridge) section is located on the bottom, and the individual electrical contacts are on the left and right of the picture. The brown line (top left) stands for $2 \mathrm{~mm}$ scale.

\subsection{Experimental set-up}

The experimental set-up proposed for the phase-locking of five emitters relies on the former rear-side extended cavity architecture demonstrated with two lasers. The laser cavity is built between a back HR mirror and the five front outputs 
of the laser devices on the tapered section side (Figure 7). The key-element of the extended cavity is a continuous phase grating diffractive optical element (DOE) $)^{19}$, which is described in $§ 3.3$. Such a phase grating placed inside the extended cavity serves two purposes: as a beam combiner to superpose the five beams from the right (rear facet of the emitter) into a single beam on-axis, and as a beam splitter to couple the back reflected light from the HR mirror (left of emitters' rear facets) into the five emitters.

The DOE couples light efficiently into the on-axis direction only when the incident beams are coherent and in the correct phase state. If the emitters are not mutually coherent, each one operates independently and is diffracted in five main diffraction orders. Since only one of these diffraction orders is along the optical axis and gets reflected back from the HR mirror, the power transmission per pass through the DOE will be of about $1 / 5$, corresponding to a round-trip transmission in the order of $(1 / 5)^{2}$ through the extended cavity. The effect is equivalent if the emitters are mutually coherent but in the wrong phase state. Consequently in both cases the laser threshold radically increases. The laser system tends to lase in the mode with the lowest losses, thus the extended cavity favors the coherent operation of the laser bar with the proper phase state for efficient combining inside the cavity.

A low-reflection glass plate is added inside the cavity for diagnostics in order to evaluate the cavity losses. The residual transmission of the HR mirror allows the measurement of the intra-cavity optical power for further analysis.

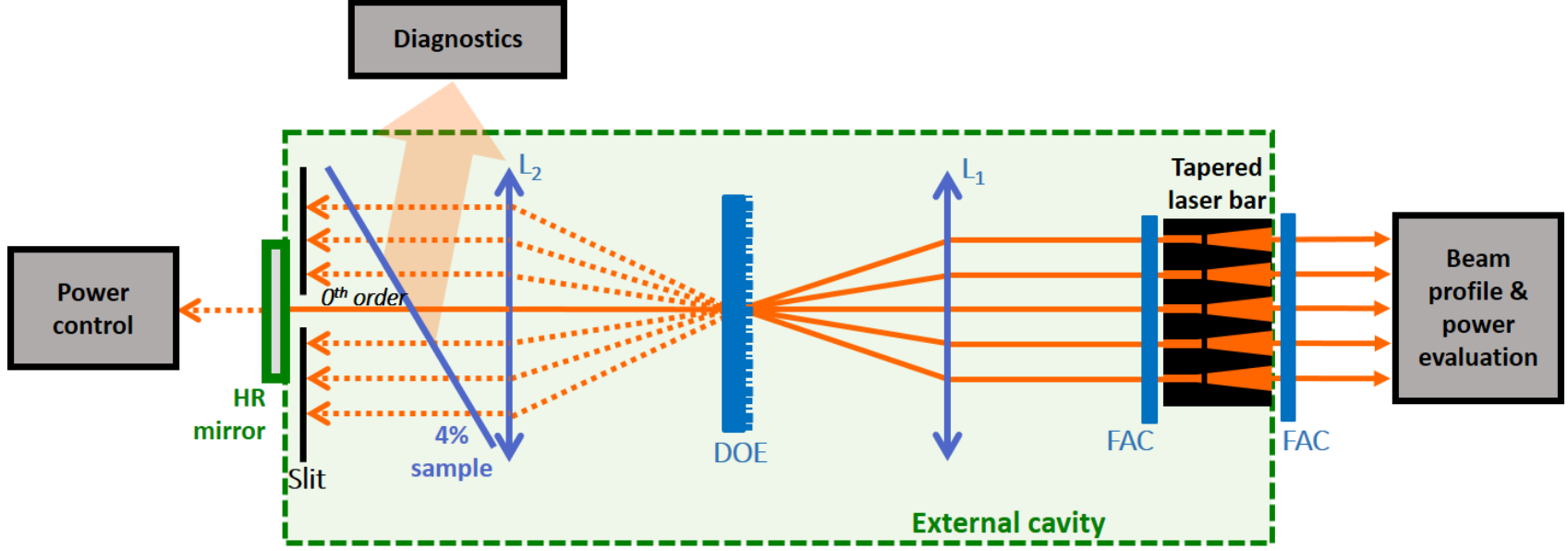

Figure 7. Experimental set-up for the phase-locking of the laser bar. The laser cavity is formed between the back HR mirror and the 5 front outputs of the laser bar. FAC: $0.6 \mathrm{~mm}$ fast axis cylindrical collimator; DOE: diffractive optical element used as beamsplitter and combiner; $\mathrm{L}_{1}, 2: 80 \mathrm{~mm}$ doublets.

\subsection{Experimental test of the DOE used as beamsplitter 1 to 5 and combiner 5 to 1}

The function of the DOE is to combine $\mathrm{N}$ beams with the correct phase state into one, or reversely to diffract one beam into $\mathrm{N}$ beams. This optical element is made with a silica plate in which a continuous phase profile was engraved and an additional AR coating added on both sides at the end of the process. The phase profile of the DOE used in this experiment has been designed numerically through an optimization procedure ${ }^{20}$ to provide a diffraction efficiency in the 5 main orders theoretically better than $98 \%$ while maintaining a diffraction uniformity along the same orders better than $30 \%$ RMS (Fig. 8, bottom). The phase state of the five main diffraction orders is $(0 \pi 000)$. 

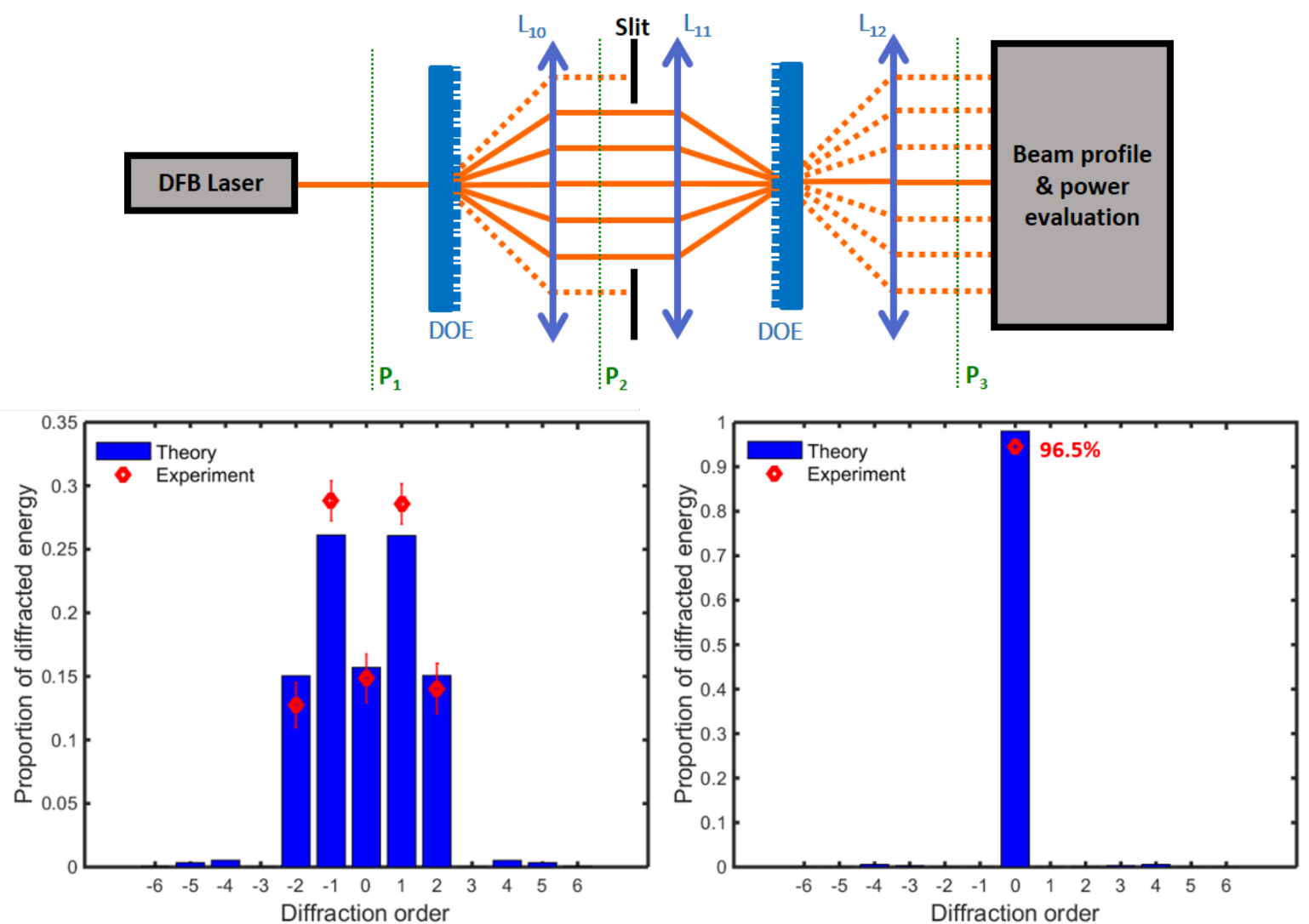

Figure 8. Experimental set-up for the validation of the DOE (top), $\mathrm{L}_{10,11,12}$ : lenses; experimental results of the diffraction by the DOE compared to simulated results for both splitting (bottom left) and combining (bottom right).

The experimental set-up dedicated to evaluate the DOE performance in both separation and combination is shown on Figure 8 (top). The laser beam from a commercial $976 \mathrm{~nm}$ distributed feedback (DFB) laser is diffracted on the DOE, the five central diffraction orders are selected using a slit, then diffracted again on another but similar DOE to recombine into one beam. The diffraction efficiency on the separation stage is defined as the ratio of the optical power diffracted in the five central orders (plane $\mathrm{P}_{2}$ ) and the optical power from the DFB laser (plane $\mathrm{P}_{1}$ ), which is measured higher than $98 \%$. The diffraction efficiency on the combining stage is defined as the ratio of the optical power diffracted in the zeroth order (plane $\mathrm{P}_{3}$ ) and the incident optical power from the five central orders (plane $\mathrm{P}_{2}$ ), measured as high as $96 \%$. The combination stage requires a very precise positioning of both the DOE and the lens $\mathrm{L}_{11}$ to avoid any residual additional phases by misalignment, as the correct phase state is needed to ensure an efficient combining. The experimental diffraction efficiency is thus in very good agreement with our theoretical simulations. A small degradation of the efficiency is observed for the combining stage, which we believe result from relative phase mismatches, coming from aberrations of the lenses at large angle or experimental misalignments.

\subsection{Coherent operation of the laser bar}

The passive phase-locking of the laser bar is observed experimentally thanks to the $4 \%$ sample glass plate inside the cavity. With an appropriate choice of currents and correct alignment of the cavity, constructive interference occurs on the DOE which we can observe through imaging of the HR mirror plane in the diagnostic arm (see Fig. 9b). The incoherent operation is differentiated from the coherent one by its distribution of light in multiple diffraction orders (see Fig. 9a). Thanks to the slit right before the HR mirror, the incoherent operation of the laser bar undergoes very high losses through one round-trip in the extended cavity. Thus the cavity favors the coherent operation of the bar and the extracted power on the front side is then significantly higher. A spectral analysis realized on each emitter separately, in both incoherent and coherent operations, reveals another interesting feature (see Fig. 10): under incoherent operation, the spectra of the different emitters exhibit unrelated contents, whereas the laser emitters are spectrally locked under 
coherent operation. The central laser wavelength under phase-locked operation is fixed by the DOE, whose spectral selectivity is evaluated to be lower than $2 \mathrm{~nm}$ FWHM in our experimental conditions.
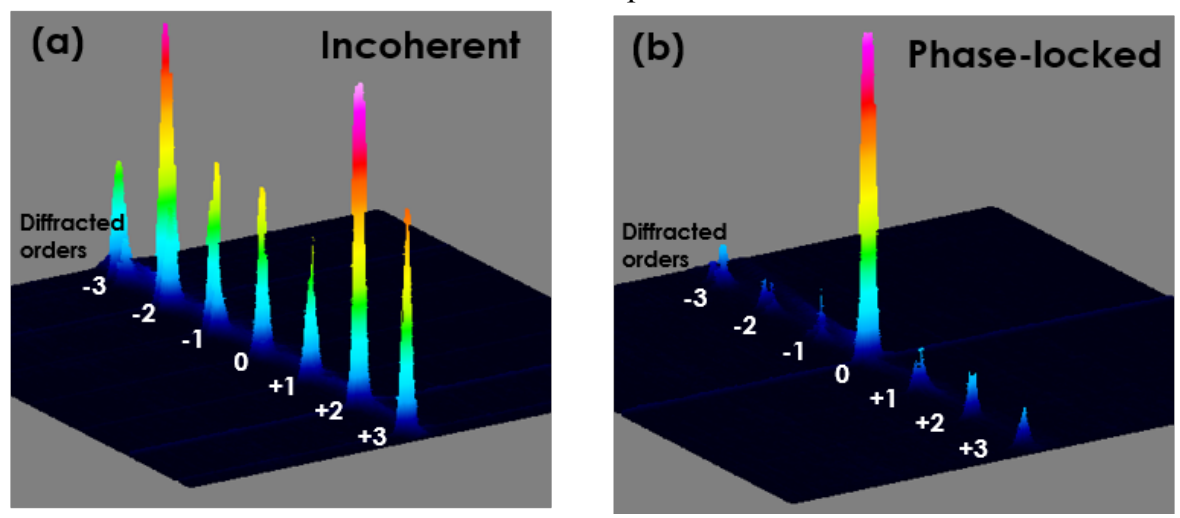

Figure 9. Near-field profiles observed on the diagnostic arm after diffraction on the DOE, for incoherent (left) and phase-locked (right) operations. Only the zeroth diffracted order is injected back into the laser cavity with the help of the slit and the HR mirror.
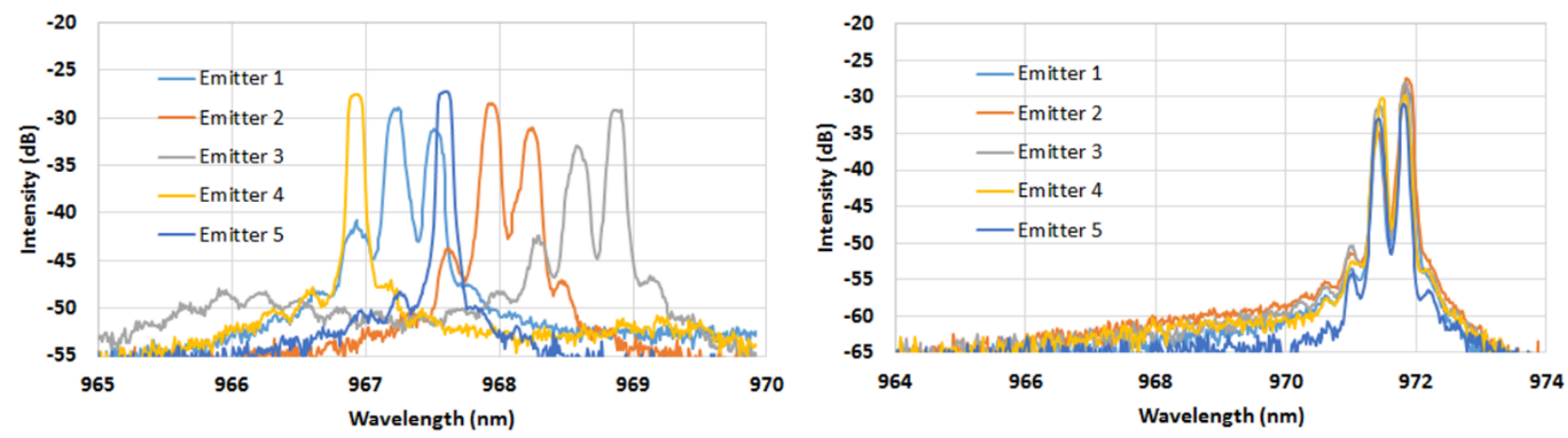

Figure 10. Experimental spectra for respectively incoherent (left) and phase-locked (right) operations.

The phase-locked operation is observed for injected currents on the tapered section up to $5 \mathrm{~A}$. This coherent operation is easily obtained at low currents and passively maintained by the cavity for several hours. For increasing tapered section currents, the coherent operation becomes more sensitive and is not systematically stable in the long term. Figure 11 shows the extracted power at the laser cavity outputs (on the front side of the emitters) as a function of the individual injected current on tapered section of each emitter, for different operation regimes of the laser bar: the phase-locked operation corresponds to the experimental set-up shown on Figure 7, while the incoherent operation corresponds to a modified external cavity (without DOE and slit) which is optimized for the overall front extracted power. The slopes of the optical power vs current curve under both coherent and incoherent operations are comparable and measured to 0.68 W.A ${ }^{-1}$, and an output power up to $12 \mathrm{~W}$ is obtained in phase-locked operation. 


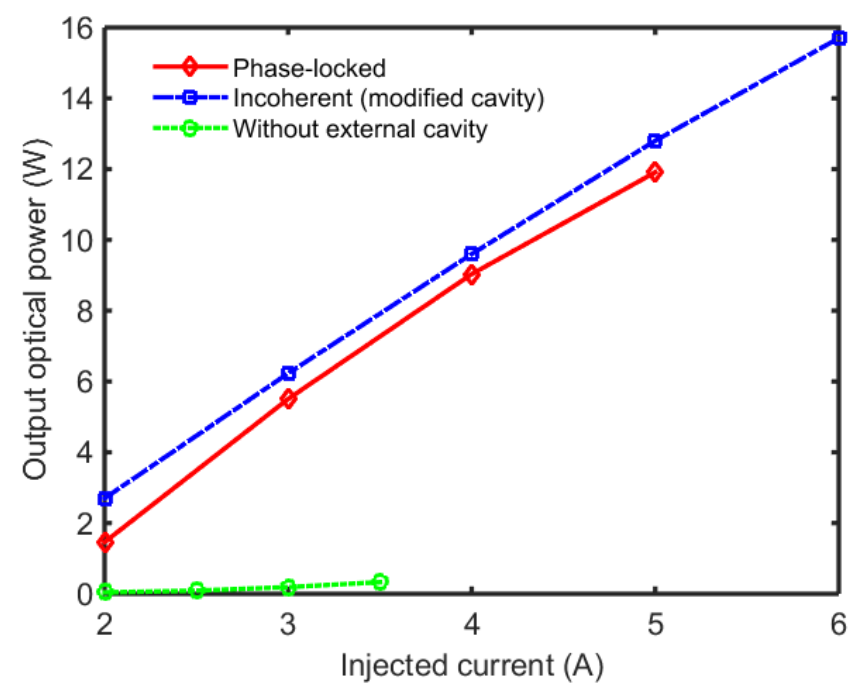

Figure 11. Extracted power on the front side of the laser bar. The experimental set-up for the phase-locked operation (red line) is described in Fig. 7, while the modified cavity for the incoherent operation (blue line) consists in the set-up without DOE and slitoptimized set-up for power extraction.

The strong mutual coherence of the emitter is confirmed by observing the interference pattern occurring on the front side of the laser bar. Thanks to the measurements of the phase shift inside the gain medium implied with a current change (shown previously on Fig 1, left), we are able to modify the accumulated phase through the emitters by changing their ridge section currents individually. A phase change of $2 \pi$ of one emitter (for one roundtrip) does not modify the phaserelationship on the intra-cavity DOE, but induces only a $\pi$ phase-shift on the front side. This feature of the extended cavity allows us to choose the relative phase between emitters on the front side by a multiple of $\pi$. Thus, the in-phase ( 0 $\left.\begin{array}{lll}0 & 0 & 0\end{array}\right)$ and out-phase $(0 \pi 0 \pi 0)$ modes of the bar can be alternately selected (Figure 12). The finesse of the interference profiles measured in the far field is 5 , which corroborates the fact that all emitters are phase-locked, with a strong mutual coherence evidenced by the high visibility $(\geq 92 \%)$.
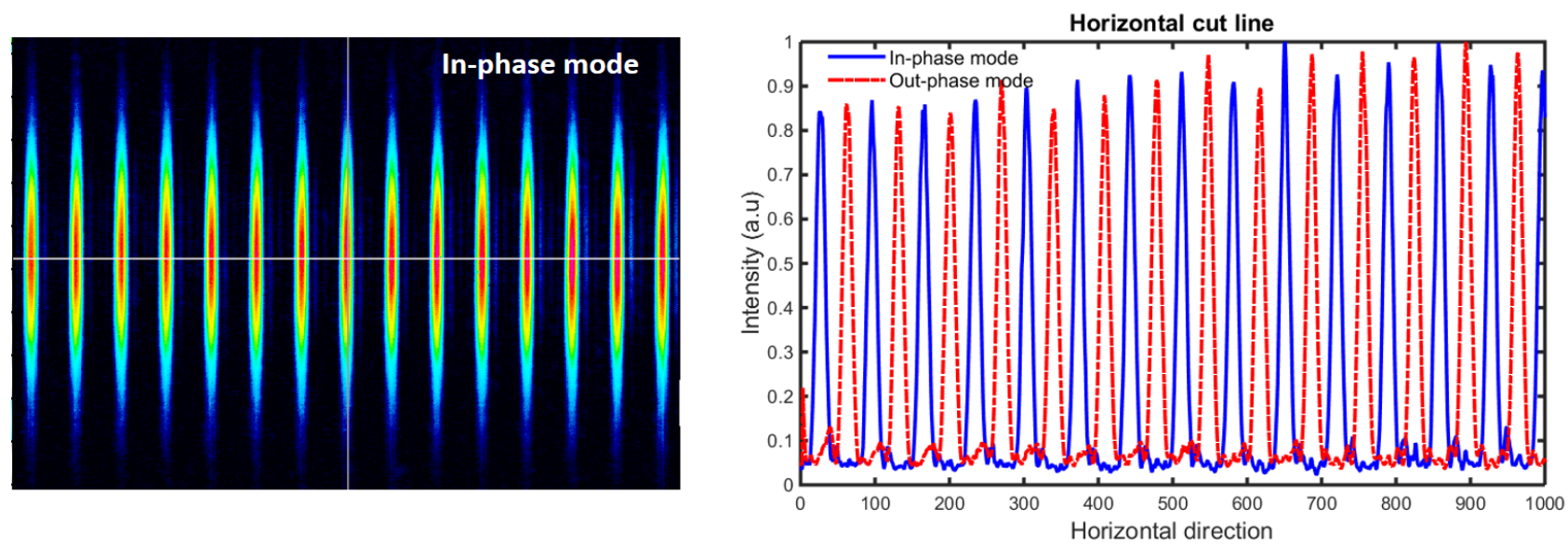

Figure 12. Front-side far-field interference pattern (left) under phase-locked operation of the laser bar into the in-phase mode. Both inphase and out-phase modes are represented on the horizontal cut line of the interference pattern (right). 


\section{CONCLUSION}

We demonstrate an innovative $\mathrm{CBC}$ architecture for diode lasers, in which the emitters are passively phase-locked by means of an extended rear-side cavity. Here, this concept is firstly implemented with two tapered lasers in a Michelsontype cavity configuration, which are then coherently combined into a single beam. An optical power of $6.7 \mathrm{~W}$ is extracted from the laser system with $>82 \%$ net combining efficiency. Secondly, the rear-side technique is applied to a 5emitter laser bar with a DOE as the combiner element, for which the phase-locked operation is obtained and studied. Up to $12 \mathrm{~W}$ are extracted under the phase-locked operation of the bar and the front combination of the beams is currently under study. The concept of this architecture is suitable for different laser types ${ }^{21,22}$. However, the benefits of such rearside resonator architecture are highlighted in this experiment by the capability to use tapered devices reaching very high brightness per emitter, and also by avoiding any drop of the coherence at high currents.

\section{REFERENCES}

[1] Köhler, B., Segref, A., Wolf, P., Unger, A., Kissel, H., Biesenbach, J., "Multi-kW high-brightness fiber coupled diode laser," Proc SPIE 8605, 86050B (2013).

[2] Huang, R. K., Chann, B., Burgess, J., Kaiman, M., Overman, R., Glenn, J. D.., Tayebati, P., "Direct diode lasers with comparable beam quality to fiber, CO2, and solid state lasers," Proc SPIE 8241, 824102 (2012).

[3] Bull, S., Ebert, G., Georges, P., Larkins, E., Lucas-Leclin, G., Brand, T., Unger, A., Traub, M., Hengesbach, S., et al., The BRIDLE Project: High Brilliance Diode Lasers for Industrial Applications, Inst. of Physics Workshop, the celebration of the 50th year of the diode laser (2012).

[4] Fan, T. Y., "Laser beam combining for high-power, high-radiance sources," IEEE J. Sel. Top. Quantum Electron. 11(3), 567-577 (2005).

[5] Brignon, A., Coherent Laser Beam Combining, John Wiley \& Sons (2013).

[6] Redmond, S. M., Creedon, K. J., Kansky, J. E., Augst, S. J., Missaggia, L. J., Connors, M. K., Huang, R. K., Chann, B., Fan, T. Y., et al., "Active coherent beam combining of diode lasers," Opt. Lett. 36(6), 999 (2011).

[7] Creedon, K. J., Redmond, S. M., Smith, G. M., Missaggia, L. J., Connors, M. K., Kansky, J. E., Fan, T. Y., Turner, G. W.., Sanchez-Rubio, A., "High efficiency coherent beam combining of semiconductor optical amplifiers," Opt. Lett. 37(23), 5006 (2012).

[8] Huang, R. K., Chann, B., Missaggia, L. J., Augst, S. J., Connors, M. K., Turner, G. W., Sanchez-Rubio, A., Donnelly, J. P., Hostetler, J. L., et al., "Coherent combination of slab-coupled optical waveguide lasers," Proc SPIE 7230, $72301 \mathrm{G}(2009)$.

[9] Paboeuf, D., Lucas-Leclin, G., Georges, P., Michel, N., Krakowski, M., Lim, J., Sujecki, S.., Larkins, E., "Narrow-line coherently combined tapered laser diodes in a Talbot external cavity with a volume Bragg grating," Appl. Phys. Lett. 93(21), 211102 (2008).

[10] Corcoran, C. J.., Durville, F., "Passive coherent combination of a diode laser array with 35 elements," Opt. Express 22(7), 8420 (2014).

[11] Montoya, J., Augst, S. J., Creedon, K., Kansky, J., Fan, T. Y.., Sanchez-Rubio, A., "External cavity beam combining of 21 semiconductor lasers using SPGD," Appl. Opt. 51(11), 1724 (2012).

[12] Bloom, G., Larat, C., Lallier, E., Carras, M.., Marcadet, X., "Coherent combining of two quantum-cascade lasers in a Michelson cavity," Opt. Lett. 35(11), 1917 (2010).

[13] Wenzel, H., Sumpf, B.., Erbert, G., "High-brightness diode lasers," Comptes Rendus Phys. 4(6), 649-661 (2003).

[14] Sabourdy, D., Kermène, V., Desfarges-Berthelemot, A., Vampouille, M.., Barthélémy, A., "Coherent combining of two Nd:YAG lasers in a Vernier-Michelson-type cavity," Appl. Phys. B 75(4-5), 503-507 (2002).

[15] Fiebig, C., Blume, G., Kaspari, C., Feise, D., Fricke, J., Matalla, M., John, W., Wenzel, H., Paschke, K., et al., "12W high-brightness single-frequency DBR tapered diode laser," Electron. Lett. 44(21), 1253-1255 (2008).

[16] Goodno, G. D., Shih, C.-C.., Rothenberg, J. E., "Perturbative analysis of coherent combining efficiency with mismatched lasers," Opt. Express 18(24), 25403 (2010).

[17] Mandel, L.., Wolf, E., Optical Coherence and Quantum Optics, Cambridge University Press (1995).

[18] Thielen, P. A., Ho, J. G., Burchman, D. A., Goodno, G. D., Rothenberg, J. E., Wickham, M. G., Flores, A., Lu, C. A., Pulford, B., et al., "Two-dimensional diffractive coherent combining of 15 fiber amplifiers into a $600 \mathrm{~W}$ beam," Opt. Lett. 37(18), 3741 (2012). 
[19] Leger, J. R., Swanson, G. J.., Veldkamp, W. B., “Coherent laser addition using binary phase gratings,” Appl. Opt. 26(20), 4391 (1987).

[20] Gale, M. T., Rossi, M., Schütz, H., Ehbets, P., Herzig, H. P.., Prongué, D., "Continuous-relief diffractive optical elements for two-dimensional array generation," Appl. Opt. 32(14), 2526 (1993).

[21] Schimmel, G., Doyen, I., Janicot, S., Ramirez, L. P., Hanna, M., Georges, P., Lucas-Leclin, G., Vilokkinen, V., Melanen, P., et al., "Separate phase-locking and coherent combining of two laser diodes in a Michelson cavity," Proc SPIE 9348, 93480P (2015).

[22] Bruesselbach, H., Jones, D. C., Mangir, M. S., Minden, M.., Rogers, J. L., "Self-organized coherence in fiber laser arrays," Opt. Lett. 30(11), 1339 (2005).

\section{Acknowledgments}

This work was supported by the European Commission within the BRIDLE program $\left(7^{\text {th }} \mathrm{FP}\right)$ under grant 314719 . We thank Katrin Paschke from FBH for detailed technical discussions. 